\title{
Thermal Conductivity of Saturated Liquid Toluene by Use of Anodized Tantalum Hot Wires at High Temperatures
}

\section{R. A. Perkins}

National Institute of Standards and Technology, Boulder, CO 80303, USA

richard.perkins@nist.gov

and

M. L. V. Ramires and C. A. Nieto de Castro

Departmento de Química e Bioquilmica and Centro de Ciência e Tecnologia de Materiais, Faculdade de Ciências da Universidade de Lisboa, Campo Grande, Ed. C1, Piso 5, 1700 Lisboa, Portugal carlos.castro@fc.ul.pt mlvr@fc.ul.pt

\author{
Absolute measurements of the thermal \\ conductivity of a distilled and dried \\ sample of toluene near saturation are \\ reported. The transient hot-wire \\ technique with an anodized tantalum hot \\ wire was used. The thermal conductivi- \\ ties were measured at temperatures from \\ $300 \mathrm{~K}$ to $550 \mathrm{~K}$ at different applied \\ power levels to assess the uncertainty with \\ which it is possible to measure liquid \\ thermal conductivity over wide temperature \\ ranges with an anodized tantalum wire. \\ The wire resistance versus temperature was \\ monitored throughout the measurements \\ to study the stability of the wire calibration. \\ The relative expanded uncertainty of the \\ resulting data at the level of 2 standard \\ deviations (coverage factor $k=2$ ) is \\ $0.5 \%$ up to $480 \mathrm{~K}$ and $1.5 \%$ between \\ $480 \mathrm{~K}$ and $550 \mathrm{~K}$, and is limited by drift \\ in the wire calibration at temperatures \\ above $450 \mathrm{~K}$. Significant thermal-radia- \\ tion effects are observed at the highest
}

temperatures. The radiation-corrected results agree well with data from transient hot-wire measurements with bare platinum hot wires as well as with data derived from thermal diffusivities obtained using light-scattering techniques.

Key words: anodized tantalum wire; liquid; thermal conductivity; thermal radiation; toluene; transient hot-wire.

Accepted: February 4, 2000

Available online: http://www.nist.gov/jres

\section{Introduction}

Saturated liquid toluene has been widely studied and is recommended by the International Union of Applied Chemistry (IUPAC) as a reference standard for thermal conductivity from $189 \mathrm{~K}$ to $360 \mathrm{~K}$ [1]. Efforts to extend this temperature range to $553 \mathrm{~K}$ have been recently reported by Ramires et al. [2]. The barriers to obtaining reliable high-temperature reference standards for thermal conductivity are a lack of data from multiple experimental techniques, and increased uncertainty due to the effects of thermal radiation. Both transient and steady-state measurement techniques for the determination of thermal conductivity are susceptible to errors due to thermal-radiative heat transfer at high temperatures since temperature gradients are imposed during the measurement, and fluids such as toluene absorb and emit the associated thermal radiation [3-5]. Even though thermal-radiation errors may be present in data from transient and steady-state techniques, agreement between radiation-corrected data from these two different techniques would provide evidence of the accuracy of the data. Unfortunately, the relative uncertainty (at the level of 2 standard deviations) of available steady-state thermal conductivity data that have been corrected for radiation exceeds $1 \%$, which is desired for the development of reference standards [2]. As a result, only data from a single transient hot-wire instrument [4] were designated as primary data during the development of the previous reference standard by Ramires et al. [2]. 
Recently, thermal diffusivity has been measured for saturated liquid toluene from $293 \mathrm{~K}$ to $523 \mathrm{~K}$ using light scattering [6]. These light-scattering data have a relative uncertainty of $2.5 \%$; and because there are no significant thermal gradients in the sample, thermal radiation errors are not present. The thermal conductivity $\lambda$ can be calculated from the thermal diffusivity $a$ by using

$$
\lambda=a \rho C_{p},
$$

where $\rho$ is the fluid mass density and $C_{p}$ is the isobaric specific heat. An accurate equation of state is available for toluene [7] to calculate $\rho$ and $C_{p}$, but uncertainties in $\mathrm{C}_{p}$ must be considered during this process. The lightscattering data were not available during the development of the previous reference standard of Ramires et al. [2].

The present measurements are made using the transient hot-wire technique as used previously [4,5]. The previous measurements were made with bare $12.7 \mu \mathrm{m}$ diameter platinum wires and were corrected for thermal radiation. The present measurements were made with anodized $25 \mu \mathrm{m}$ diameter tantalum wires. Anodized tantalum wires have the advantage of being electrically insulated from the fluid under study. This anodized coating allows measurements of electrically conducting fluids such as water. The highest temperature at which toluene has been previously studied with an anodized-tantalum hot-wire instrument is $370 \mathrm{~K}$ [8]. The present measurements extend the temperature range at which anodized tantalum hot wires have been used to measure thermal conductivity from $370 \mathrm{~K}$ to $550 \mathrm{~K}$. The use of anodized-tantalum hot wires allows some evaluation of the reliability of the thermal radiative correction for absorbing media since the anodized tantalum wires have a different emissivity (that of tantalum pentoxide) than those of the previous platinum wires, and the diameter of the tantalum wire is twice that of the previous platinum wires.

\section{Experimental}

The transient hot-wire technique is widely recognized as an accurate method to measure the thermal conductivity and thermal diffusivity of fluids. The present measurements are absolute and require only knowledge of the geometry of the hot wires, the applied power, the resistance-versus-temperature characteristics of the wires, and time. The ideal working equation is based on the heat transfer from an infinitely long line source into an infinite medium. The temperature rise of the fluid at the surface of the wire, where $r=r_{0}$, is given [9] by

$$
\Delta T_{\text {ideal }}\left(r_{0}, t\right)=\frac{q}{4 \pi \lambda} \ln \left(\frac{4 a t}{r_{0}^{2} C}\right)=\frac{q}{4 \pi \lambda} \ln (t)+\frac{q}{4 \pi \lambda} \ln \left(\frac{4 a}{r_{0}^{2} C}\right)
$$

where $q$ is the power divided by the length of the wire, $t$ is the elapsed time, and $C=\mathrm{e}^{\gamma}=1.781 \ldots$ is the exponential of Euler's constant. The ideal temperature rise of the wire is linear with respect to the logarithm of elapsed time, as shown in Eq. (2). The thermal conductivity is obtained from the slope, and the thermal diffusivity is obtained from the intercept using linear regression [10]. The temperature associated with a given thermal conductivity data point is given by

$$
T_{r}=T_{0}+0.5\left(\Delta T_{\text {initial }}+\Delta T_{\text {final }}\right),
$$

where $\Delta T_{\text {initial }}$ and $\Delta T_{\text {final }}$ are the temperature rise at the start time and the end time of the linear region selected for the regression. The thermal diffusivity is associated with the initial cell temperature $T_{0}$ and is obtained from a calibrated reference-standard platinum resistance thermometer (PRT). All temperatures in this work are reported according to the 1990 International Temperature Scale (ITS 90), and all uncertainties are expanded uncertainties at the level of 2 standard deviations (coverage factor $k=2,95 \%$ level of confidence).

The experimental cell is designed to approximate this ideal model as closely as possible. There are, however, a number of corrections that account for deviations between the ideal line-source solution and the actual experimental heat transfer. The ideal temperature rise is obtained by adding a number of corrections $\delta T \mathrm{i}$ to the experimental temperature rise according to

$$
\Delta T_{\text {ideal }}=\Delta T_{\text {experimental }}+\sum_{i} \delta T_{i}
$$

These temperature-rise corrections are described in detail for our case of a coated wire in Refs. [3,11,12]. Our implementation of the corrections follows these references with the following exceptions. The compression work correction $\delta T_{3}$ and the radial convection correction $\delta T_{4}$ are set to zero following the recommendations of Assael et al. [13]. The thermal-radiation correction $\delta T_{5}$ is described for absorbing fluids by Nieto de Castro et al. [5].

The data-acquisition system used in this work has been described previously [4], and consists of a microcomputer with a 16 bit analog-to-digital converter, three digital voltmeters, a digital power supply, and a Wheatstone bridge which contains two hot wires in opposing legs of the bridge. The two hot wires have different lengths, and the Wheatstone bridge, which is initially 
balanced, subtracts the resistance change of the short hot wire from the resistance change of the long hot wire. Thus, if both wires are immersed in the same fluid, the bridge response behaves as for a finite length (the difference between the wire lengths) of an infinitely long wire and the end effects arising from axial conduction are eliminated. Heating voltage is applied to the wires through the Wheatstone bridge, and the bridge imbalance is measured in 250 equal time increments. The total time for the measurements may be varied from $1 \mathrm{~s}$ to $40 \mathrm{~s}$, allowing one to verify that the data are obtained prior to the onset of convection. The computer checks the bridge balance prior to each experiment and records the temperature of the reference thermometer and the resistance of each hot wire for calibration purposes. The cell temperature is measured with an uncertainty of $1 \mathrm{mK}$ by use of a current source and a standard resistor in series with the reference-standard PRT. The cell pressure is measured with a quartz pressure transducer from $0 \mathrm{MPa}$ to $70 \mathrm{MPa}$ with an uncertainty of $0.007 \mathrm{MPa}$.

\subsection{Hot-Wire Cell}

The hot-wire cell used in these measurements was designed for measurements on corrosive solutions at temperatures from $300 \mathrm{~K}$ to $550 \mathrm{~K}$ at pressures up to $70 \mathrm{MPa}$. The design of the pressure vessel and temperature control system is the same as for our previous hightemperature cells [4], so it will only be briefly described here. The pressure vessel and the internal components of the hot-wire cell are constructed of nickel alloy UNS-N10276, which is particularly resistant to halides and halide salts. The cell contains long and short hot wires located within long and short cavities with diameters of $9.5 \mathrm{~mm}$. The total volume of the cell and supporting pressure system is relatively small, about $50 \mathrm{~cm}^{3}$, to facilitate measurements on scarce or hazardous materials. There are separate voltage and current leads to each end of each hot wire to eliminate the effects of lead resistance during the calibration process. All electrical connections in the cell are spot welded. In the assembly of the tantalum hot wires $(25.4 \mu \mathrm{m}$ diameter), polytetrafluoroethane-insulated nickel/chromium alloy wire $(254 \mu \mathrm{m}$ diameter) and polyimide-insulated platinum ( $76 \mu \mathrm{m}$ diameter) wire were used to make electrical connections inside the pressure system. The connections were welded, then all the bare leads were coated three times with a polyimide/polytetrafluoroethane resin, and the assembly was baked at $550 \mathrm{~K}$ for several hours. The baking both cured the polymer resin and annealed the tantalum hot wires from their initial hard-drawn condition. The tantalum hot wires were then anodized in aqueous citric acid with up to $50 \mathrm{~V}$ to produce a film of tantalum pentoxide with an estimated thickness of $70 \mathrm{~nm}$. Although the temperature control and pressure systems are rated to $750 \mathrm{~K}$, the upper operating temperature of the present tantalum hot-wire cell assembly is limited to $550 \mathrm{~K}$ because of the melting point of polytetrafluoroethane used to electrically insulate the lead wires.

\subsection{Sample Purification}

The toluene sample used in these measurements was prepared from spectroscopic-grade toluene. The toluene was further purified by distillation over calcium hydride. Calcium hydride reacts irreversibly with any water in the sample to form calcium hydroxide precipitate, which remains in the distillation flask. The principal impurity is benzene, which has a lower boiling temperature, so the initial condensate is discarded. The sample used for measurement is then collected when the inlet to the condenser is stable at the boiling temperature of toluene. The purified sample was analyzed by gas chromatography and found to have less than $50 \mathrm{ng} / \mathrm{g}$ of benzene and less than $100 \mathrm{ng} / \mathrm{g}$ of water. The sample preparation procedure was the same as in our previous measurements [4] using bare platinum hot wires.

\subsection{Wire Calibration}

Platinum is preferred for use in resistance thermometry when it is properly annealed and is free from stress because its resistance is very stable for prolonged periods of time. Tantalum has not been used widely for resistance thermometry, so the stability of its resistance must be carefully examined and characterized. The situation is further complicated since the present tantalum wires have been anodized to form a protective layer of tantalum pentoxide. The electrical resistivity of tantalum from $273 \mathrm{~K}$ to $1273 \mathrm{~K}$ has been shown to increase in proportion to the concentration of oxygen in the sample [14]. Since the concentration of oxygen is not uniform through the wire's cross section, there is a possibility that oxygen from the anodized layer might diffuse into the bulk tantalum wire and alter its resistance. Any oxygen-diffusion process would be enhanced at higher temperatures. The present system is ideally suited for characterizing the anodized tantalum wires since the wire resistance is measured for each wire, along with the temperature from the reference PRT and the pressure from the quartz pressure transducer, during the balance cycle for each measurement. The instrument also maintains a record of the time and date of each measurement to allow examination of the stability of the wire calibration. Since the present measurements were made along the saturation line of toluene, at pressures less than $3.3 \mathrm{MPa}$, there is not enough pressure range to allow characterization of the 
pressure dependence. The electrical resistivity of tantalum is known to decrease in proportion to the pressure on the sample [15].

To eliminate uncertainty due to the resistance of the lead wires, there are separate current-supply and voltage-sensing leads to each end of each hot wire. The resistance is measured during the balance cycle by measuring the voltage drop across standard resistors in each leg of the Wheatstone bridge containing the hot wires, together with the voltage drop across each hot wire. The measured value is the average of five readings with a forward current of about $0.3 \mathrm{~mA}$ and five readings with the current reversed. This process minimizes uncertainty due to thermoelectric voltages at weld junctions and electrical connectors. The uncertainty of the resistance measurement is about $0.003 \Omega$. The measurements were made first with temperature increasing from $300 \mathrm{~K}$ to $550 \mathrm{~K}$. Then, measurements were made at $550 \mathrm{~K}$ in the morning and evening for a period of 4 days. Finally, measurements were made with temperature decreasing from $550 \mathrm{~K}$ to $300 \mathrm{~K}$ so that hysteresis effects could be examined. The electrical resistance of the long $(188.08 \mathrm{~mm})$ and short $(49.07 \mathrm{~mm})$ hot wires are shown in Fig. 1 during this temperature cycle. It is apparent in the figure that the resistances of both the long and short hot wires increased with the elapsed time at $550 \mathrm{~K}$. Although the resistance of the long wire increased more than that of the short wire, the increase was not in proportion to the wire lengths, as would be expected if the process were uniform over the entire length of each wire.

Since both the long and short hot wires come from the same sample of tantalum wire, the resistance of each wire should scale with the length of each wire. This is a requirement for use of these wires in the transient hot-wire experiment. To insure uniform heat generation over the length of each hot wire, the resistance divided by the length of the long and short hot wires respectfully must be very nearly equal. It is desirable to characterize both the uniformity of the power generation in both hot wires and the adequacy of compensation for the end effects by using two wires in different arms of the measuring bridge. This can be measured by taking the ratio $\sigma_{\mathrm{lw}} / \sigma_{\mathrm{sw}}$ between the resistance divided by the length of the long wire, $\sigma_{\mathrm{lw}}=R_{\mathrm{sw}} / L_{\mathrm{sw}}$, and the resistance divided by the length of the short wire, $\sigma_{\mathrm{sw}}=R_{\mathrm{sw}} / L_{\mathrm{sw}}$, where $R$ is the wire resistance, $L$ is the wire length, and the subscripts lw and sw designate the long wire and short wire, respectively. Following a previous recommendation by Kestin and Wakeham [16], $\sigma_{\mathrm{lw}} / \sigma_{\mathrm{sw}}$ must not deviate from unity by more than $2 \%$, in order to assume a correct end-effect compensation with nearly identical wires. With the definition

$$
\Delta \sigma=100\left(\frac{\sigma_{\mathrm{lw}}}{\sigma_{\mathrm{sw}}}-1\right)
$$

Figure 2 shows this percentage difference as a function of elapsed time. It can be seen that this deviation was quite stable and nearly zero during the experiments at increasing temperature from $300 \mathrm{~K}$ to the start of the $550 \mathrm{~K}$ isotherm but began to drift as the resistance of both wires increased. There was a decrease of $2 \%$ by the end of the $550 \mathrm{~K}$ isotherm. This behavior is compatible with previous calculations by Kestin and Wakeham [16].

However, the increase in resistance during the 4 days at $550 \mathrm{~K}$ is quite dramatic and unexpected, based on our previous experience with pure platinum hot wires [4]. With pure platinum the normal behavior is a slight decrease in resistance due to annealing and stress release in the hot wires if the wires have not been at this temperature recently. Figure 2 clearly shows that the increase in resistance was not consistent with wire length. Thus, resistance increases at the welds must be considered a possibility. The only weld locations that can contribute to the measured resistance during a four terminal measurement occur where the ends of the $25 \mu \mathrm{m}$ diameter tantalum wires are joined to the $254 \mu \mathrm{m}$ diameter nickel-chromium alloy lead wires. Each hot wire has two welds which could potentially contribute to the measured resistance, but it is not possible to separate contributions due to the welds from those due to changes in the wires. The use of all-tantalum lead wires should be examined in the future to see whether this resistance increases within the welds or within the wires themselves.

Based on Fig. 1 and the requirement that $\Delta \sigma \leq 2 \%$, it was decided that only the data at increasing temperatures, including the first few hours at $550 \mathrm{~K}$, should be considered for the wire-resistance calibration. The wire's resistance was fit to a quadratic polynomial in temperature of the form $R(T)=A_{1}+A_{2} T+A_{3} T^{2}$ over four regions, $300 \mathrm{~K}$ to $450 \mathrm{~K}, 300 \mathrm{~K}$ to $480 \mathrm{~K}, 300 \mathrm{~K}$ to $515 \mathrm{~K}$, and $300 \mathrm{~K}$ to $550 \mathrm{~K}$. The results of these fits are given in Table 1 . The resistance of a pure metal such as tantalum is known to be well approximated by such a quadratic expression [17], and the sign of $A_{3}$ should be small and negative over this temperature range. Table 1 shows that the sign of the quadratic coefficients change if the resistance data above $480 \mathrm{~K}$ are included in the fits. This is a good indication that the increase in resistance with time becomes significant at temperatures above $480 \mathrm{~K}$.

Given that the increase in resistance with time is significant at $515 \mathrm{~K}$, the best possible calibration must be determined, and the influence of uncertainty in the 


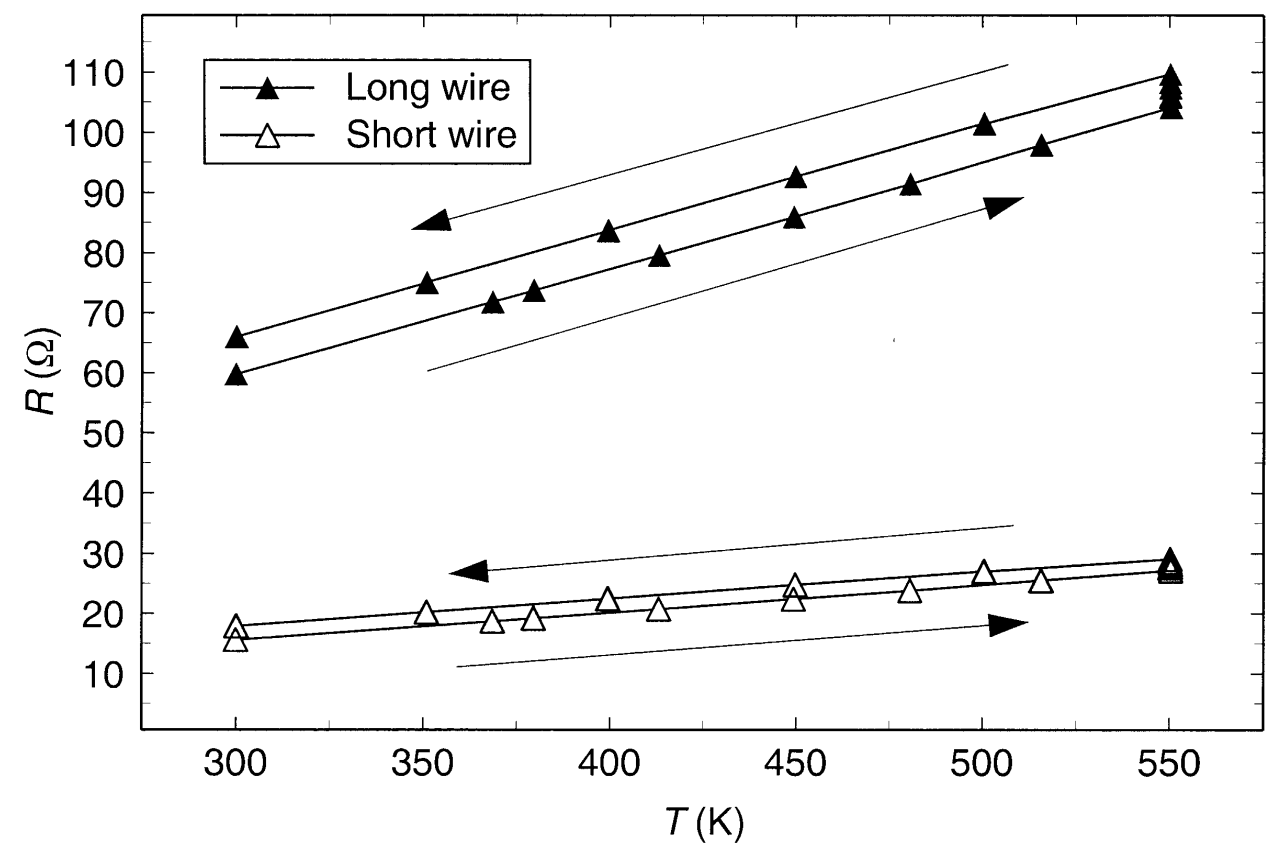

Fig. 1. Resistance of anodized tantalum hot wires as a function of temperature. Arrows indicate the sequence of elapsed time (first increasing then decreasing temperature).

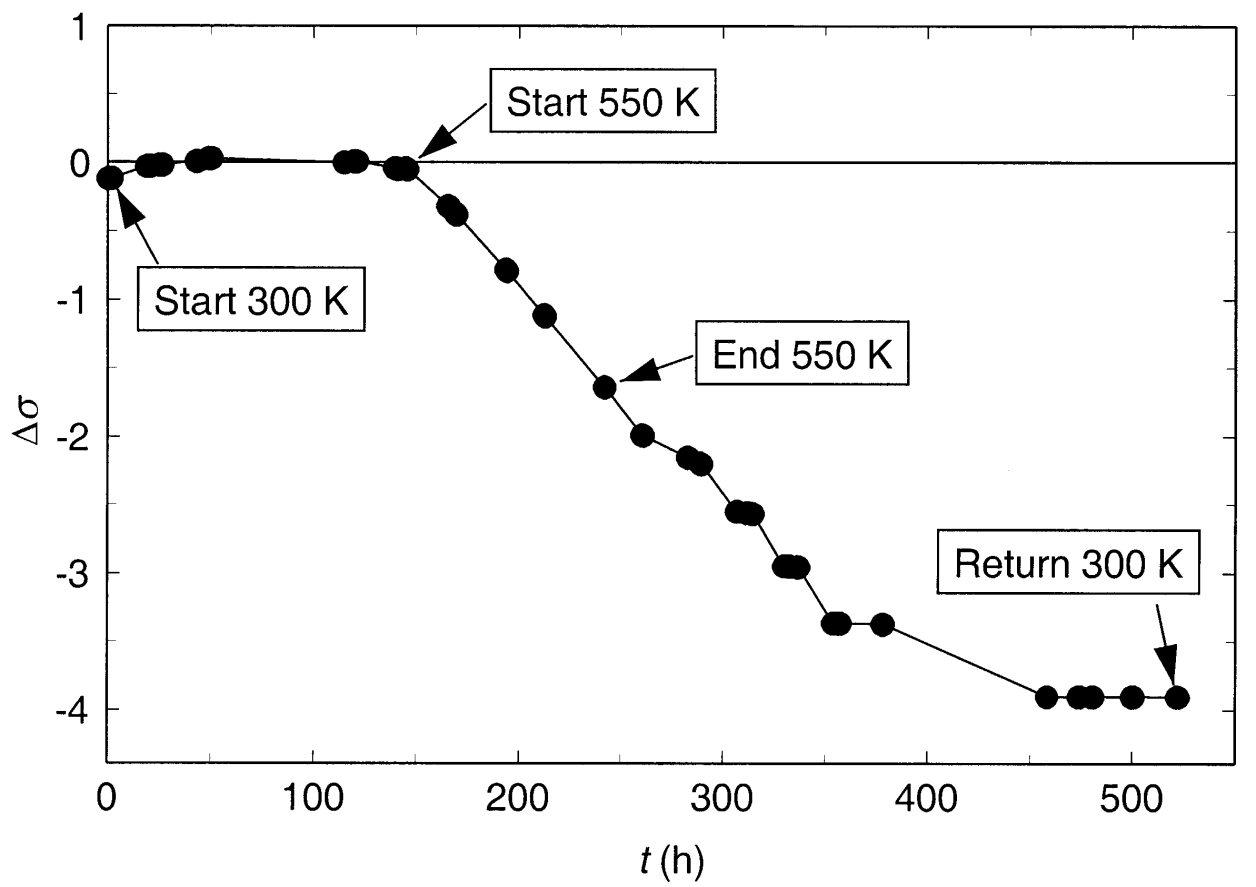

Fig. 2. Plot of the quantity $\Delta \sigma$ as a function of time, where $\Delta \sigma=100\left(\sigma_{\mathrm{lw}} / \sigma_{\mathrm{sw}}-1\right)$ with $\sigma_{\mathrm{lw}}=R_{\mathrm{lw}} / L_{\mathrm{lw}}$ and $\sigma_{\mathrm{sw}}=R_{\mathrm{sw}} / L_{\mathrm{sw}}$. Here $R$ is the wire resistance, $L$ is the wire length, and the subscripts lw and sw designate the long wire and short wire, respectively. 
Table 1. Calibration coefficients for the anodized tantalum hot wires

\begin{tabular}{lclc}
\hline \hline Temp./Wire & $\begin{array}{c}A_{1} \\
(\Omega)\end{array}$ & \multicolumn{1}{c}{$\begin{array}{c}A_{2} \\
\left(\Omega \mathrm{K}^{-1}\right)\end{array}$} & $\begin{array}{c}A_{3} \\
\left(\Omega \mathrm{K}^{-2}\right)\end{array}$ \\
\hline $450 \mathrm{~K} /$ Long & 6.42577 & 0.179655 & $-0.595598 \times 10^{-5}$ \\
$450 \mathrm{~K} /$ Short & 1.81164 & $0.463252 \times 10^{-1}$ & $-0.102980 \times 10^{-5}$ \\
$480 \mathrm{~K} /$ Long & 6.64231 & 0.178426 & $-0.424702 \times 10^{-5}$ \\
$480 \mathrm{~K} /$ Short & 1.90216 & $0.458112 \times 10^{-1}$ & $-0.315381 \times 10^{-6}$ \\
$515 \mathrm{~K} /$ Long & 9.25254 & 0.164042 & $0.150564 \times 10^{-4}$ \\
$515 \mathrm{~K} /$ Short & 2.66652 & $0.415993 \times 10^{-1}$ & $0.533726 \times 10^{-5}$ \\
$550 \mathrm{~K} /$ Long & 9.47142 & 0.162877 & $0.165577 \times 10^{-4}$ \\
$550 \mathrm{~K} /$ Short & 2.69778 & $0.414329 \times 10^{-1}$ & $0.555170 \times 10^{-5}$ \\
\hline
\end{tabular}

Given that the increase in resistance with time is significant at $515 \mathrm{~K}$, the best possible calibration must be determined, and the influence of uncertainty in the calibration on the thermal conductivity results must be assessed. The rise in temperature at any elapsed time during a measurement is obtained from the change in resistance of the hot wires using the derivative of the calibration curve for resistance versus temperature:

$$
\Delta T=\frac{\Delta R}{\left.\frac{\partial R}{\partial T}\right|_{T}}=\frac{\Delta R}{\left(A_{2}+2 A_{3} T\right)} .
$$

Thus, the uncertainty of the wire's temperature rise, and of the measured thermal conductivity, is directly related to the uncertainty of the derivative of wire resistance with respect to temperature. This resistance derivative divided by length is plotted as a function of temperature in Fig. 3, which shows the effect of the change in sign of $\mathrm{A}_{3}$ when resistance data at temperatures above $480 \mathrm{~K}$ are included in the fit. Based on data below $480 \mathrm{~K}$, where there is confidence that the calibration is stable, the resistance derivative decreases by $1 \%$ for a temperature increase of $180 \mathrm{~K}$. Since this temperature dependence is quite small, and the region of extrapolation $(480 \mathrm{~K}$ to $550 \mathrm{~K})$ is less than half this temperature range, it is anticipated that extrapolation errors should be less than $1 \%$ if only data below $480 \mathrm{~K}$ are used for the calibration. If resistance data from temperatures above $480 \mathrm{~K}$ are used in the calibration, the thermal conductivity results at $550 \mathrm{~K}$ will be about $4 \%$ higher at $550 \mathrm{~K}$ and results at $300 \mathrm{~K}$ will be $2 \%$ too low. Based on these considerations, the wire calibration from $300 \mathrm{~K}$ to $480 \mathrm{~K}$ is used in the subsequent data analysis, with the estimate that the relative uncertainty of the measured thermal conductivity increases above $480 \mathrm{~K}$ by the additive amount of $1.0 \%$ due to extrapolation in the wire calibration.

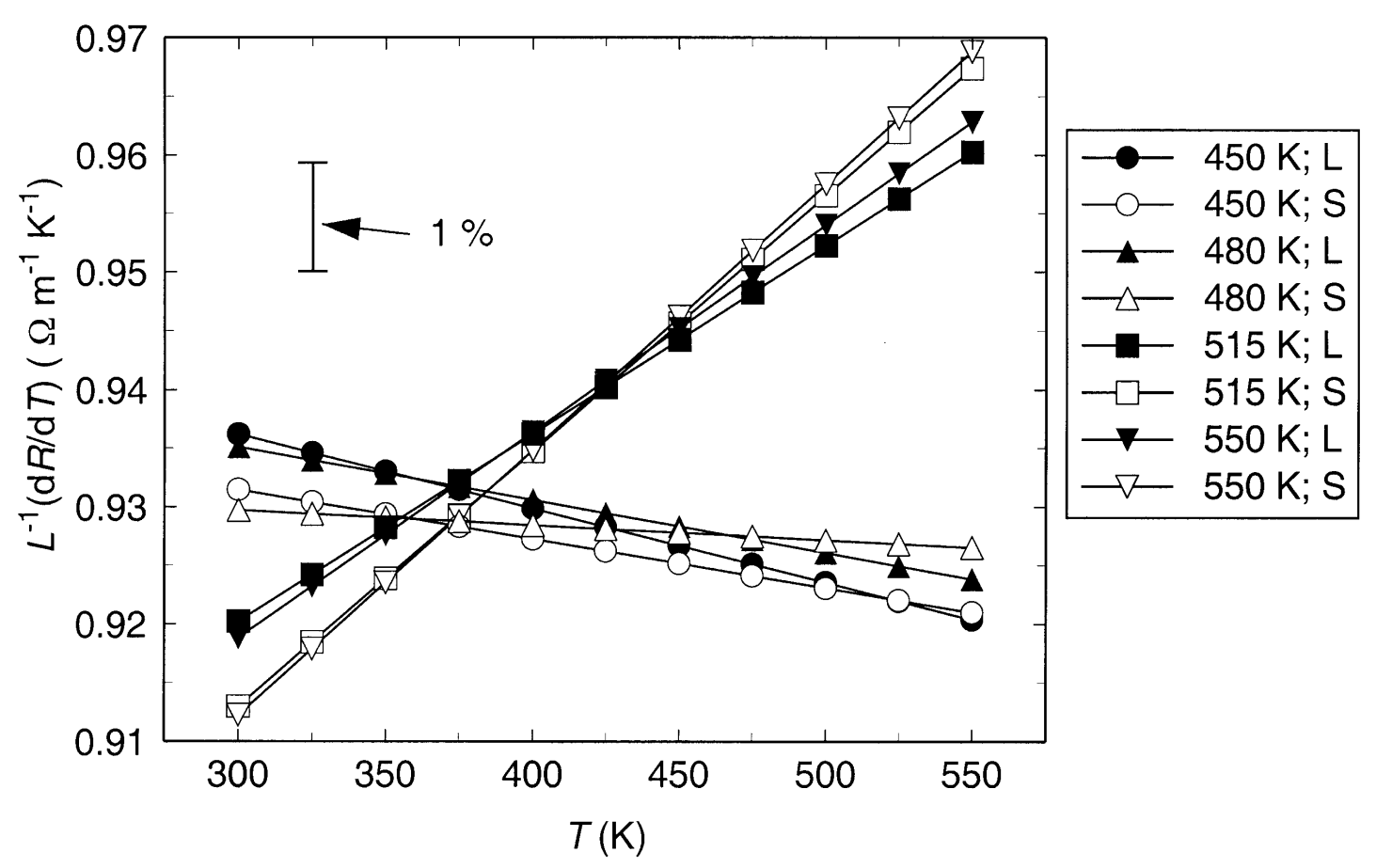

Fig. 3. Values of $\frac{\mathrm{d} R}{\mathrm{~d} T}$ divided by the length of anodized tantalum wire. 


\section{Results}

The thermal conductivity results for the purified toluene sample are given in Table 2 and are shown in Fig. 4. The results in Table 2 have been corrected for thermal radiation, as were our previous results using bare platinum hot wires [4], and with the same empirical optical parameters that were found for the fluid using the previous platinum hot wires [5]. Since the power divided by length was not equal for the long and short wires after the isotherm at $550 \mathrm{~K}$, as shown in Fig. 2, the data from the decreasing temperature portion of the temperature cycle are not reported. There are 184 thermal conductivity data points at temperatures from $300 \mathrm{~K}$ to $550 \mathrm{~K}$.

\subsection{Repeatability at High Temperatures}

The isotherm at $550 \mathrm{~K}$ is quite interesting since it includes several replications, with a wide range of power levels, over the course of four days. During this time, the resistance of the long hot wire increased by $5.6 \Omega$ and that of the short hot wire increased by $1.9 \Omega$. In addition, since this increase in resistance was not proportional to the wire lengths, the power generation of the two wires differed by up to $1.6 \%$. Dispite these complications, there seems to be little additional scatter in the thermal conductivity results measured during this 4 day period. In Fig. 5, deviations between the results for the $550 \mathrm{~K}$ isotherm are plotted relative to the reference standard of Ramires et al. [2] as a function of applied power level at $550 \mathrm{~K}$. The mean deviation of the data (solid line) is $1.45 \%$ higher than the earlier reference standard, and the scatter (dashed lines) is $\pm 0.6 \%$ at the level of $95 \%$ confidence. No trend is noted with respect to time throughout this four-day period. Convection in the sample occurs at shorter times for experiments with higher powers and correspondingly larger temperature rises. Consistency between thermal conductivity results at different power levels is considered a good indication that there was no significant convection during the measurements and that compensation for the wire's end effects was achieved. Data at power levels above $0.5 \mathrm{Wm}^{-1}$ appear to have some influence due to the onset of convection since this isotherm is relatively close to the critical point of toluene at $593.95 \mathrm{~K}$. The contribution of convection on the apparent thermal conductivity appears to be less than $0.5 \%$ at even the highest power levels.

\subsection{Uncertainty Assessment}

The contribution of thermal radiation to measurements of the thermal conductivity of fluids such as toluene has been a topic of debate for many years. An empirical technique has been described for correcting for thermal radiation in transient hot-wire measurements [5]. Empirical optical parameters have been reported for toluene [5] at these same conditions based on measurements with platinum hot wires $12.7 \mu \mathrm{m}$ in diameter. If this radiation correction is valid, then these same optical parameters should apply to the present case of a tantalum wire $25 \mu \mathrm{m}$ in diameter. The radiation correction assumes that thermal emission from the wire is small compared to emission from the expanding thermal front in the fluid, so there should be little effect due to changing the emissivity of the wire material. The contribution of thermal radiation is insignificant at $300 \mathrm{~K}$, increases as $T^{3}$ and is estimated to be $3 \%$ at $550 \mathrm{~K}$. The experimental thermal conductivity data with and without this radiative correction are shown in Fig. 4.

Deviations between the reference standard of Ramires et al. [2] and the present data are shown in Fig. 6. High-temperature data sets from light scattering [6] as well as transient hot-wire experiments using bare platinum $[4,18]$ and anodized tantalum $[8]$ hot wires are also compared in this figure. The data of Kraft et al. [6] were obtained from thermal diffusivities by light scattering using the equation of state of Goodwin [7]. These light-scattering data are free from uncertainty due to thermal radiation but are believed to have a relative uncertainty of $2.5 \%$ in thermal diffusivity. The uncertainty in the thermal conductivity obtained from the expression $\lambda=a \rho C_{p}$ is estimated to vary between $3 \%$ and $4.2 \%$ since the uncertainties in $\rho$ and $C_{p}$ from the equation of state [7] are $0.2 \%$ for $\rho$ and varies from $0.2 \%$ to $3 \%$ for $C_{p}$. The thermal conductivity obtained from light scattering is offset from the direct thermal conductivity measurements by about $2 \%$ to $3 \%$, but agreement is still within the combined uncertainty of the data sets. It is unlikely that the offset is due to thermal radiation since it is nearly constant and thermal-radiative errors should increase as $T^{3}$.

No systematic difference between transient hot-wire measurements using bare and anodized tantalum hot wires is apparent in Fig. 6. The present tantalum hotwire data are lower than the other transient data $[4,8,18]$ at $300 \mathrm{~K}$ by about $2 \%$. This is partly due to larger cell fluctuations of temperature in the furnace containing the hot-wire cell near ambient temperature. The remainder of this difference is likely due to the drift in calibration of the tantalum hot wires at elevated temperatures, as shown in Fig. 3. The uncertainty of the present tantalum measurements are evaluated as $1 \%$ at $300 \mathrm{~K}, 0.5 \%$ from $369 \mathrm{~K}$ to $480 \mathrm{~K}$, and $1.5 \%$ from $480 \mathrm{~K}$ to $550 \mathrm{~K}$. The transient hot-wire data using platinum or anodized tantalum wires agree within their combined uncertainties over the entire temperature range, with the exception of the data of Yamada et al. 


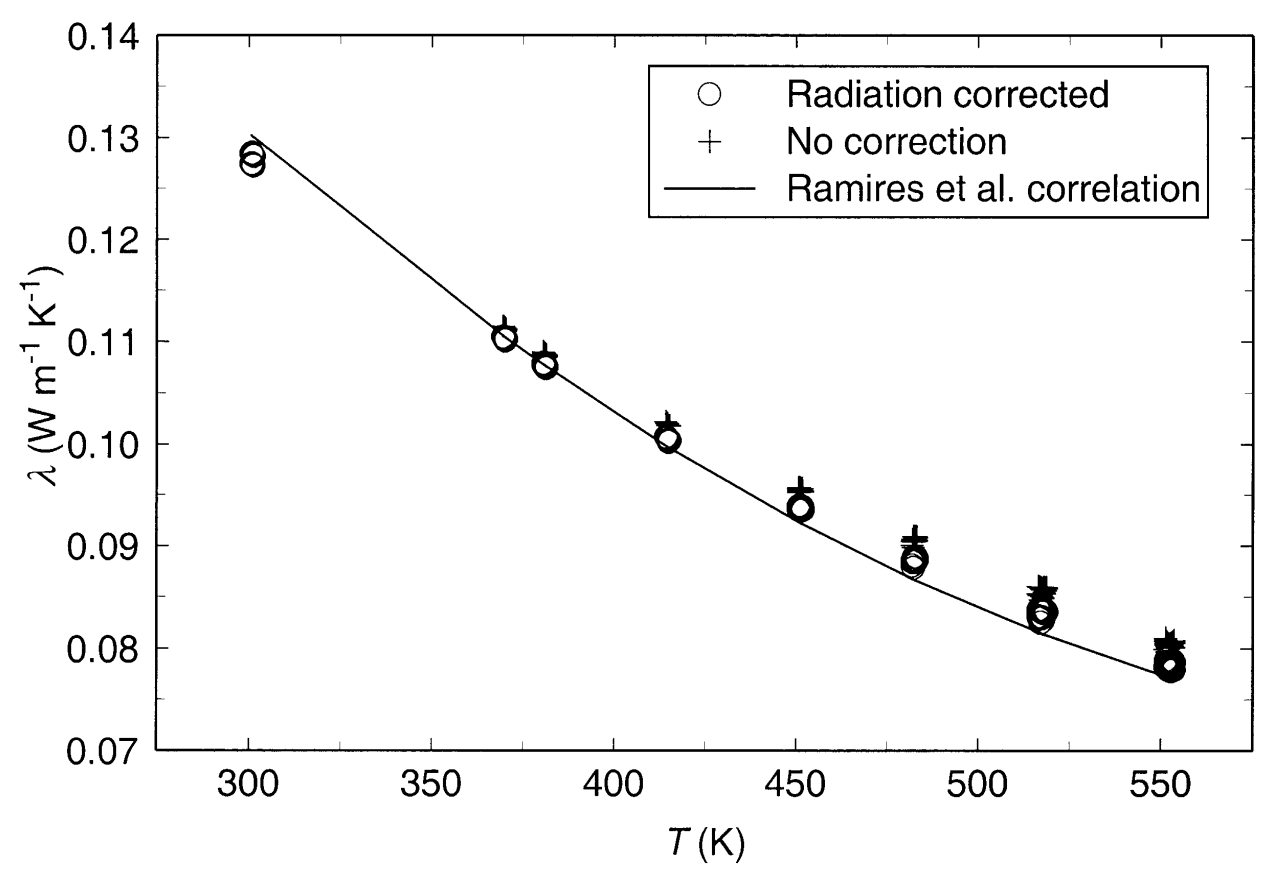

Fig. 4. Thermal conductivity of saturated liquid toluene as a function of temperature. The correction for thermal radiation increases with $T^{3}$ to about $3 \%$ at $550 \mathrm{~K}$.

Table 2. Thermal conductivity of toluene near the saturated liquid line

\begin{tabular}{|c|c|c|c|c|c|c|c|}
\hline $\begin{array}{c}T \\
(\mathrm{~K})\end{array}$ & $\begin{array}{c}p \\
(\mathrm{MPa})\end{array}$ & $\begin{array}{c}\lambda \\
\left(\mathrm{W} \mathrm{m}^{-1} \mathrm{~K}^{-1}\right)\end{array}$ & $\begin{array}{c}q \\
\left(\mathrm{~W} \mathrm{~m}^{-1}\right)\end{array}$ & $\begin{array}{c}T \\
(\mathrm{~K})\end{array}$ & $\begin{array}{c}p \\
(\mathrm{MPa})\end{array}$ & $\begin{array}{c}\lambda \\
\left(\mathrm{W} \mathrm{m}^{-1} \mathrm{~K}^{-1}\right)\end{array}$ & $\begin{array}{c}q \\
\left(\mathrm{~W} \mathrm{~m}^{-1}\right)\end{array}$ \\
\hline 301.248 & 3.246 & 0.12818 & 0.34440 & 381.226 & 0.092 & 0.10747 & 0.39108 \\
\hline 301.150 & 3.252 & 0.12820 & 0.31730 & 381.078 & 0.088 & 0.10742 & 0.35911 \\
\hline 301.051 & 3.253 & 0.12824 & 0.29135 & 380.929 & 0.087 & 0.10776 & 0.32851 \\
\hline 300.950 & 3.258 & 0.12827 & 0.26653 & 380.804 & 0.084 & 0.10769 & 0.29929 \\
\hline 300.856 & 3.260 & 0.12840 & 0.24281 & 380.672 & 0.081 & 0.10771 & 0.27143 \\
\hline 300.762 & 3.260 & 0.12852 & 0.22020 & 380.551 & 0.079 & 0.10781 & 0.24493 \\
\hline 300.676 & 3.263 & 0.12849 & 0.19870 & 380.429 & 0.078 & 0.10773 & 0.21980 \\
\hline 300.595 & 3.266 & 0.12845 & 0.17831 & 381.304 & 0.293 & 0.10764 & 0.42455 \\
\hline 301.231 & 0.077 & 0.12725 & 0.34442 & 381.166 & 0.292 & 0.10750 & 0.39111 \\
\hline 301.134 & 0.075 & 0.12723 & 0.31730 & 381.024 & 0.290 & 0.10753 & 0.35906 \\
\hline 301.030 & 0.067 & 0.12735 & 0.29132 & 380.889 & 0.290 & 0.10776 & 0.32846 \\
\hline 300.934 & 0.059 & 0.12728 & 0.26649 & 380.759 & 0.288 & 0.10777 & 0.29925 \\
\hline 300.834 & 0.053 & 0.12736 & 0.24278 & 380.620 & 0.288 & 0.10785 & 0.27138 \\
\hline 300.745 & 0.047 & 0.12735 & 0.22017 & 380.509 & 0.286 & 0.10781 & 0.24489 \\
\hline 300.661 & 0.044 & 0.12749 & 0.19868 & 380.391 & 0.285 & 0.10794 & 0.21976 \\
\hline 300.580 & 0.040 & 0.12744 & 0.17829 & 414.154 & 0.272 & 0.10076 & 0.22435 \\
\hline 370.214 & 0.101 & 0.11017 & 0.41357 & 414.288 & 0.271 & 0.10071 & 0.25066 \\
\hline 370.078 & 0.101 & 0.11014 & 0.38102 & 414.416 & 0.271 & 0.10064 & 0.27846 \\
\hline 369.941 & 0.101 & 0.11013 & 0.34985 & 414.559 & 0.270 & 0.10060 & 0.30776 \\
\hline 369.820 & 0.101 & 0.11033 & 0.32003 & 414.709 & 0.270 & 0.10043 & 0.33856 \\
\hline 369.698 & 0.101 & 0.11038 & 0.29155 & 414.858 & 0.269 & 0.10023 & 0.37081 \\
\hline 369.578 & 0.101 & 0.11048 & 0.26441 & 415.036 & 0.269 & 0.10038 & 0.40456 \\
\hline 369.467 & 0.101 & 0.11049 & 0.23859 & 415.201 & 0.269 & 0.10037 & 0.43975 \\
\hline 369.368 & 0.101 & 0.11051 & 0.21411 & 451.768 & 0.522 & 0.09351 & 0.49427 \\
\hline 370.250 & 0.324 & 0.11024 & 0.41358 & 451.575 & 0.522 & 0.09369 & 0.45548 \\
\hline 370.115 & 0.310 & 0.11021 & 0.38103 & 451.393 & 0.522 & 0.09373 & 0.41830 \\
\hline 369.982 & 0.297 & 0.11011 & 0.34982 & 451.223 & 0.522 & 0.09381 & 0.38268 \\
\hline 369.859 & 0.286 & 0.11039 & 0.32001 & 451.044 & 0.522 & 0.09377 & 0.34864 \\
\hline 369.739 & 0.276 & 0.11039 & 0.29152 & 450.874 & 0.522 & 0.09376 & 0.31618 \\
\hline 369.611 & 0.267 & 0.11043 & 0.26438 & 450.720 & 0.521 & 0.09371 & 0.28532 \\
\hline 369.500 & 0.259 & 0.11043 & 0.23857 & 450.573 & 0.521 & 0.09368 & 0.25605 \\
\hline 369.393 & 0.252 & 0.11050 & 0.21409 & 451.732 & 0.859 & 0.09387 & 0.49447 \\
\hline 381.373 & 0.093 & 0.10752 & 0.42440 & 451.539 & 0.858 & 0.09374 & 0.45550 \\
\hline
\end{tabular}


Table 2. Thermal conductivity of toluene near the saturated liquid line - Continued

\begin{tabular}{|c|c|c|c|c|c|c|c|}
\hline $\begin{array}{c}T \\
(\mathrm{~K})\end{array}$ & $\begin{array}{c}p \\
(\mathrm{MPa})\end{array}$ & $\begin{array}{c} \\
\left(\mathrm{W} \mathrm{m}^{-1} \mathrm{~K}^{-1}\right)\end{array}$ & $\begin{array}{c}q \\
\left(\mathrm{~W} \mathrm{~m}^{-1}\right)\end{array}$ & $\begin{array}{c}T \\
(\mathrm{~K})\end{array}$ & $\begin{array}{c}p \\
(\mathrm{MPa})\end{array}$ & $\begin{array}{c}\lambda \\
\left(\mathrm{W} \mathrm{m}^{-1} \mathrm{~K}^{-1}\right)\end{array}$ & $\begin{array}{c}q \\
\left(\mathrm{~W} \mathrm{~m}^{-1}\right)\end{array}$ \\
\hline 451.364 & 0.858 & 0.09390 & 0.41824 & 552.555 & 2.567 & 0.07798 & 0.38226 \\
\hline 451.172 & 0.857 & 0.09345 & 0.38264 & 552.367 & 2.567 & 0.07805 & 0.34496 \\
\hline 451.017 & 0.857 & 0.09360 & 0.34860 & 552.182 & 2.566 & 0.07822 & 0.30959 \\
\hline 450.855 & 0.854 & 0.09390 & 0.31616 & 552.004 & 2.565 & 0.07792 & 0.27613 \\
\hline 450.711 & 0.855 & 0.09391 & 0.28531 & 551.852 & 2.563 & 0.07839 & 0.24458 \\
\hline 450.566 & 0.855 & 0.09393 & 0.25603 & 551.688 & 2.562 & 0.07845 & 0.21495 \\
\hline 451.752 & 0.802 & 0.09362 & 0.49480 & 551.547 & 2.562 & 0.07854 & 0.18725 \\
\hline 451.567 & 0.802 & 0.09391 & 0.45583 & 553.467 & 2.628 & 0.07857 & 0.60640 \\
\hline 451.382 & 0.802 & 0.09387 & 0.41852 & 553.200 & 2.632 & 0.07848 & 0.55867 \\
\hline 451.213 & 0.803 & 0.09379 & 0.38285 & 552.981 & 2.635 & 0.07835 & 0.51297 \\
\hline 451.037 & 0.804 & 0.09372 & 0.34877 & 552.771 & 2.637 & 0.07842 & 0.46924 \\
\hline 450.876 & 0.803 & 0.09402 & 0.31629 & 552.550 & 2.640 & 0.07825 & 0.42750 \\
\hline 450.711 & 0.804 & 0.09353 & 0.28541 & 552.365 & 2.641 & 0.07822 & 0.38773 \\
\hline 450.566 & 0.805 & 0.09372 & 0.25614 & 552.165 & 2.643 & 0.07815 & 0.34990 \\
\hline 483.230 & 0.922 & 0.08856 & 0.52551 & 551.991 & 2.645 & 0.07816 & 0.31401 \\
\hline 483.013 & 0.917 & 0.08851 & 0.48420 & 551.816 & 2.646 & 0.07832 & 0.28008 \\
\hline 482.805 & 0.913 & 0.08846 & 0.44467 & 551.656 & 2.647 & 0.07839 & 0.24807 \\
\hline 482.598 & 0.910 & 0.08876 & 0.40678 & 551.501 & 2.648 & 0.07838 & 0.21803 \\
\hline 482.413 & 0.908 & 0.08845 & 0.37060 & 551.367 & 2.649 & 0.07870 & 0.18992 \\
\hline 482.234 & 0.906 & 0.08786 & 0.33612 & 553.540 & 2.657 & 0.07863 & 0.60776 \\
\hline 482.064 & 0.905 & 0.08864 & 0.30331 & 553.293 & 2.658 & 0.07843 & 0.55988 \\
\hline 481.906 & 0.903 & 0.08808 & 0.27220 & 553.057 & 2.658 & 0.07834 & 0.51402 \\
\hline 483.220 & 1.574 & 0.08883 & 0.52569 & 552.830 & 2.659 & 0.07833 & 0.47020 \\
\hline 483.019 & 1.574 & 0.08893 & 0.48421 & 552.604 & 2.659 & 0.07827 & 0.42839 \\
\hline 482.808 & 1.574 & 0.08862 & 0.44455 & 552.393 & 2.659 & 0.07829 & 0.38853 \\
\hline 482.614 & 1.572 & 0.08899 & 0.40668 & 552.193 & 2.659 & 0.07815 & 0.35062 \\
\hline 482.433 & 1.572 & 0.08858 & 0.37053 & 552.001 & 2.659 & 0.07819 & 0.31468 \\
\hline 482.263 & 1.571 & 0.08894 & 0.33608 & 551.814 & 2.659 & 0.07822 & 0.28069 \\
\hline 482.086 & 1.571 & 0.08852 & 0.30329 & 551.652 & 2.658 & 0.07839 & 0.24862 \\
\hline 481.934 & 1.572 & 0.08845 & 0.27217 & 551.492 & 2.659 & 0.07858 & 0.21850 \\
\hline 518.628 & 1.534 & 0.08352 & 0.56232 & 551.342 & 2.659 & 0.07888 & 0.19033 \\
\hline 518.393 & 1.533 & 0.08338 & 0.51802 & 553.646 & 2.635 & 0.07865 & 0.61486 \\
\hline 518.162 & 1.533 & 0.08324 & 0.47568 & 553.388 & 2.635 & 0.07854 & 0.56649 \\
\hline 518.032 & 1.533 & 0.08308 & 0.43519 & 553.139 & 2.635 & 0.07837 & 0.52016 \\
\hline 517.748 & 1.533 & 0.08275 & 0.39648 & 552.896 & 2.634 & 0.07832 & 0.47586 \\
\hline 517.546 & 1.532 & 0.08305 & 0.35960 & 552.668 & 2.635 & 0.07828 & 0.43354 \\
\hline 517.348 & 1.532 & 0.08293 & 0.32450 & 552.445 & 2.634 & 0.07812 & 0.39321 \\
\hline 517.179 & 1.532 & 0.08296 & 0.29120 & 552.240 & 2.634 & 0.07802 & 0.35485 \\
\hline 517.010 & 1.532 & 0.08251 & 0.25972 & 552.047 & 2.634 & 0.07808 & 0.31845 \\
\hline 516.792 & 1.532 & 0.08303 & 0.23005 & 551.852 & 2.634 & 0.07820 & 0.28403 \\
\hline 516.638 & 1.532 & 0.08292 & 0.20218 & 551.684 & 2.634 & 0.07819 & 0.25158 \\
\hline 516.501 & 1.532 & 0.08314 & 0.17611 & 551.517 & 2.634 & 0.07832 & 0.22111 \\
\hline 518.578 & 2.143 & 0.08367 & 0.56245 & 551.367 & 2.633 & 0.07857 & 0.19261 \\
\hline 518.347 & 2.144 & 0.08366 & 0.51804 & 553.163 & 2.619 & 0.07843 & 0.61933 \\
\hline 518.147 & 2.143 & 0.08357 & 0.47563 & 553.346 & 2.619 & 0.07882 & 0.57103 \\
\hline 517.928 & 2.142 & 0.08373 & 0.43512 & 553.083 & 2.620 & 0.07842 & 0.52429 \\
\hline 517.670 & 2.142 & 0.08364 & 0.39647 & 552.840 & 2.620 & 0.07831 & 0.47962 \\
\hline 517.482 & 2.141 & 0.08350 & 0.35957 & 552.612 & 2.620 & 0.07834 & 0.43695 \\
\hline 517.275 & 2.142 & 0.08363 & 0.32448 & 552.388 & 2.620 & 0.07817 & 0.39628 \\
\hline 517.094 & 2.142 & 0.08367 & 0.29119 & 552.169 & 2.621 & 0.07789 & 0.35763 \\
\hline 516.915 & 2.142 & 0.08374 & 0.25973 & 551.974 & 2.621 & 0.07802 & 0.32095 \\
\hline 516.764 & 2.143 & 0.08371 & 0.23005 & 551.784 & 2.621 & 0.07820 & 0.28626 \\
\hline 516.606 & 2.143 & 0.08374 & 0.20218 & 551.607 & 2.621 & 0.07822 & 0.25355 \\
\hline 516.467 & 2.143 & 0.08380 & 0.17610 & 551.436 & 2.622 & 0.07824 & 0.22284 \\
\hline 553.594 & 2.565 & 0.07794 & 0.59749 & 551.285 & 2.622 & 0.07820 & 0.19411 \\
\hline 553.379 & 2.567 & 0.07793 & 0.55059 & 552.155 & 2.642 & 0.07809 & 0.36233 \\
\hline 553.178 & 2.566 & 0.07804 & 0.50564 & 551.949 & 2.642 & 0.07798 & 0.32512 \\
\hline 552.967 & 2.567 & 0.07810 & 0.46260 & 551.762 & 2.642 & 0.07815 & 0.28994 \\
\hline 552.740 & 2.567 & 0.07783 & 0.42146 & 551.579 & 2.642 & 0.07821 & 0.25680 \\
\hline
\end{tabular}




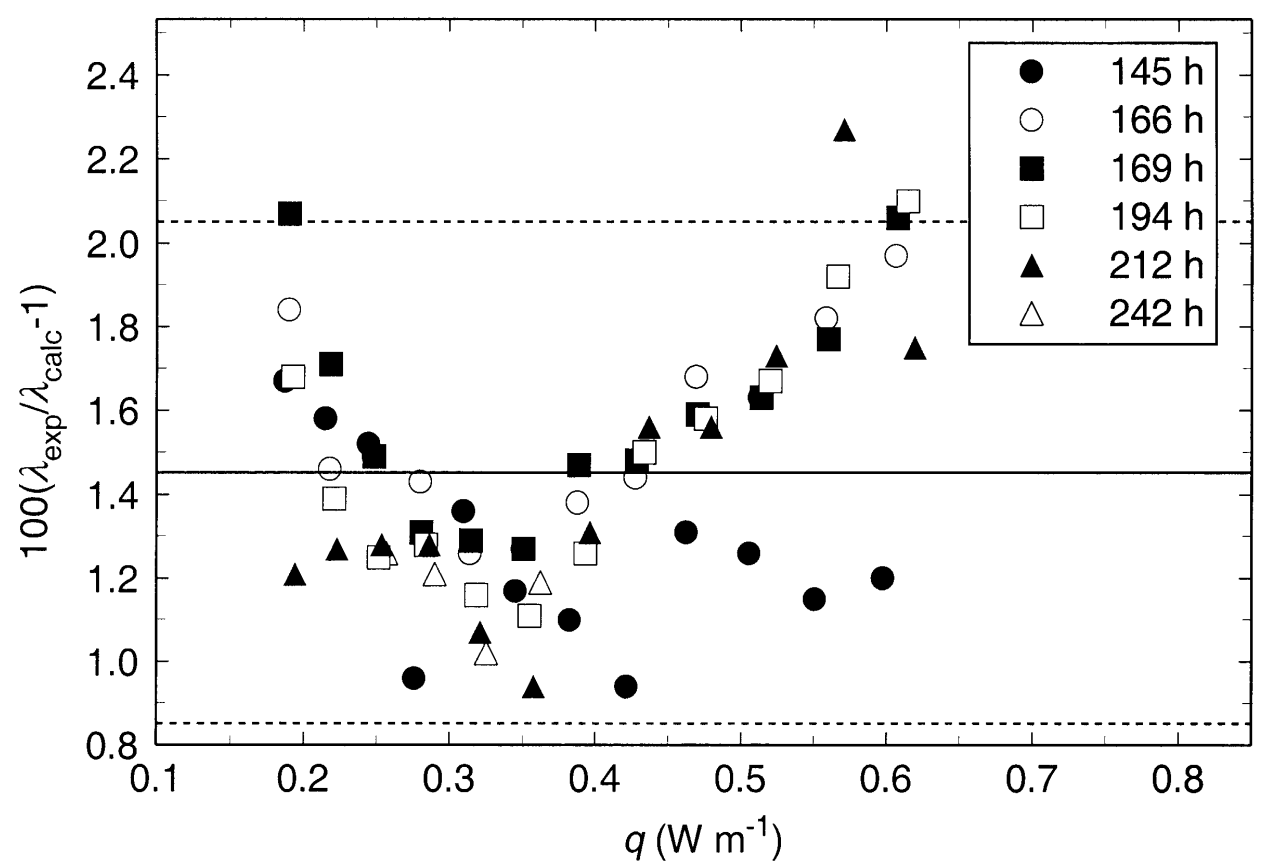

Fig. 5. Thermal conductivity of saturated liquid toluene as a function of applied power near $550 \mathrm{~K}$. Different plot symbols designate the average elapsed time of the experiment series. The mean deviation of the data (solid line) is $1.45 \%$ higher than the earlier reference standard, and the scatter (dashed lines) is $\pm 0.6 \%$ at the level of $95 \%$ confidence.

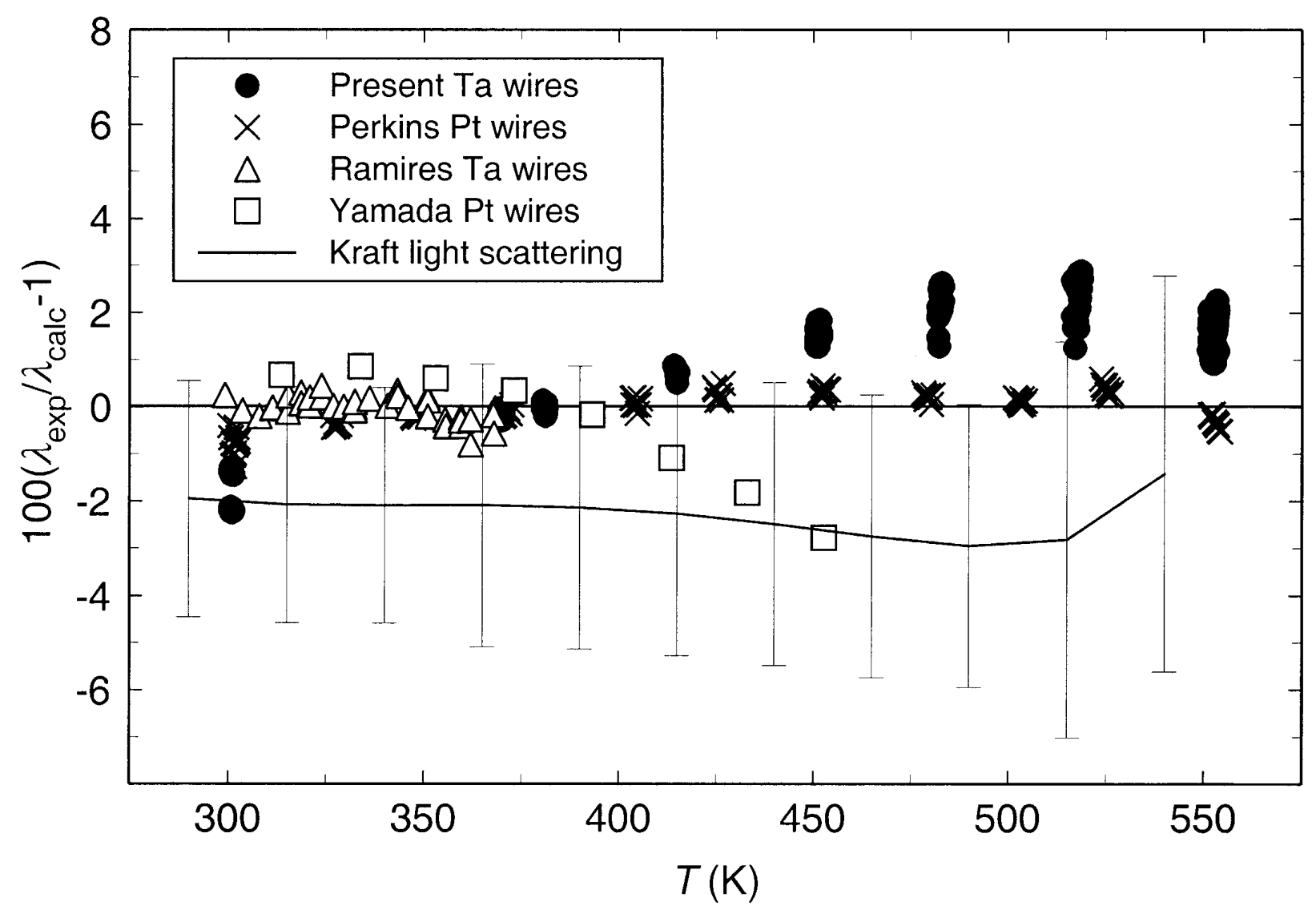

Fig. 6. Deviations between the thermal conductivity data, $[4,6,8,18]$ and present work, and the reference standard of Ramires et al. [2]. 
[18] at temperatures above $400 \mathrm{~K}$. The purity of the toluene sample used in this case [18] was stated to be $99.7 \%$ by the supplier. The purity of the toluene sample used in the light scattering study [6] was stated to be $99.9 \%$ by the supplier. The transient hot-wire data of Perkins et al. [4] and Ramires et al. [8] were made on purified samples of toluene as described in the present work.

\section{Conclusions}

The present measurements demonstrate that anodized-tantalum hot wires can be used to make absolute measurements of the thermal conductivity a liquid from $300 \mathrm{~K}$ to $550 \mathrm{~K}$. Previous studies with anodized-tantalum hot wires have been limited to temperatures below $370 \mathrm{~K}$ [8]. The present transient hot-wire measurements using anodized-tantalum hot wires have a larger uncertainty in the temperature extremes than our previous measurements using bare tantalum hot wires [4] over the same temperature range. This is primarily due to drift in the resistance calibration of the anodized tantalum hot wires at high temperatures. Use of tantalum lead wires may reduce or eliminate this problem in the future and allow accurate measurements of the fluid thermal diffusivity. It was also noted during the experiments that convection occurs earlier, and at lower power levels, as the wire diameter increases. Thus, experiments must be done with lower levels of applied power (smaller temperature rises) when the larger tantalum hot wires are used.

The big advantage of anodized-tantalum hot wires is for measurement of electrically conducting fluids, and this is not a problem in the case of toluene. The anodized tantalum hot wires have a geometry and emissivity different from those of platinum, so the present measurements support the validity of the thermal-radiation correction for absorbing fluids [5]. There is no significant temperature trend in deviations between the present radiation-corrected thermal-conductivity data and thermal-diffusivity data derived from light-scattering measurements of thermal diffusivity. This again supports the validity of the radiation correction [5], since the contribution of thermal radiation is expected to increase with $T^{3}$.

\section{Acknowledgments}

We gratefully acknowledge the financial support of this work by the United States Department of Energy, Division of Engineering and Geosciences, Office of Basic Energy Sciences, through DE-AI02-96ER14597. Both M. L. V. Ramires and C. A. Nieto de Castro thank the Faculty of Sciences of the University of Lisbon for a leave of absence and the Physical and
Chemical Properties Division of the National Institute of Standards and Technology for support as guest researchers. M. L. V. Ramires also thanks JNICT, Portugal, for an additional grant.

\section{References}

[1] C. A. Nieto de Castro, S. F. Y. Li, A. Nagashima, R. D. Trengove, and W. A. Wakeham, J. Phys. Chem. Ref. Data 15, 1078 (1986).

[2] M. L. V. Ramires, C. A. Nieto de Castro, and R. A. Perkins, High Temp. High Press. 25, 269 (1993).

[3] W. A. Wakeham, A. Nagashima, and J. V. Sengers, eds., Measurement of the Transport Properties of Fluids, Experimental Thermodynamics Volume III, Blackwell Scientific Publications, Oxford (1991).

[4] R. A. Perkins, H. M. Roder, and C. A. Nieto de Castro, J. Res. Natl. Inst. Stand. Technol. 96, 247 (1991).

[5] C. A. Nieto de Castro, R. A. Perkins, and H. M. Roder, Int. J. Thermophys. 12, 985 (1991).

[6] K. Kraft, M. Matos Lopes, and A. Leipertz, Int. J. Thermophys. 16, 423 (1995).

[7] R. D. Goodwin, J. Phys. Chem. Ref. Data 18, 1565 (1989).

[8] M. L. V. Ramires, J. M. N. A. Fareleira, C. A. Nieto de Castro, M. Dix, and W. A. Wakeham, Int. J. Thermophys. 14, 1119 (1993).

[9] H. S. Carslaw and J. C. Jaeger, Conduction of heat in solids, 2nd ed., Oxford University Press, London (1959).

[10] C. A. Nieto de Castro, B. Taxis, H. M. Roder, and W. A. Wakeham, Int. J. Thermophys. 9, 293 (1992).

[11] J. J. Healy, J. J. de Groot, and J. Kestin, Physica 82C, 392 (1976).

[12] Y. Nagasaka and A. Nagashima, J. Phys. E: Sci. Instrum. 14, 1435 (1981).

[13] M. J. Assael, L. Karagiannidis, S. M. Richardson, and W. A. Wakeham, Int. J. Thermophys. 13, 223 (1992).

[14] E. Gebhardt and H. D. Seghezzi, Z. Metallk. 48, 430 (1957).

[15] P. W. Bridgman, Proc. Amer. Acad. Arts Sci. 81, 165 (1952).

[16] J. Kestin, and W. A. Wakeham, Physica 92A, 102 (1978).

[17] Y. S. Touloukian, ed., Thermophysical properties of high temperature solid materials-Volume 1: Elements, Macmillan, New York, (1967).

[18] T. Yamada, T. Yaguchi, Y. Nagasaka, and A. Nagashima, High Temp. High Press. 25, 513 (1993).

About the authors: Richard A. Perkins is a chemical engineer in the Physical and Chemical Properties Division of the NIST Chemical Science and Technology Laboratory. Maria L. V. Ramires is also a chemical engineer and Auxiliary Professor at the Faculdade de Ciências da Universidade de Lisboa, and was a Guest Researcher at NIST. Carlos A. Nieto de Castro is a chemical engineer, director of the Centro de Ciência e Tecnologia de Materiais and of the Laboratory of Metrology and Testing of the Instituto de Ciencia Aplicada e Tecnologia, Faculdade de Ciências da Universidade de Lisboa, and a Guest Researcher at NIST. The National Institute of Standards and Technology is an agency of the Technology Administration, U.S. Department of Commerce. 